\title{
Origin of heart rate variability and turbulence: an appraisal of autonomic modulation of cardiovascular function
}

\author{
Federico Lombardi ${ }^{1}$ * and Phyllis K. Stein ${ }^{2}$ \\ ${ }^{1}$ Cardiologia, Dipartimento di Medicina, Chirurgia e Odontoiatria, Ospedale San Paolo, University of Milan, Milan, Italy \\ ${ }^{2}$ Heart Rate Variability Laboratory, Washington University School of Medicine, St. Louis, MO, USA
}

Edited by:

Heikki Veli Huikuri, University of Oulu,

Finland

\section{Reviewed by:}

Heikki Veli Huikuri, University of Oulu, Finland

Antti M. Kiviniemi, Verve, Finland

*Correspondence:

Federico Lombardi, Cardiologia, Dipartimento di Medicina, Chirurgia e Odontoiatria, Ospedale San Paolo, University of Milan, Via di Rudini 8 , 20142 Milan, Italy.

e-mail: federico.lombardi@unimi.it
Heart period constantly changes on a beat to beat basis, due to autonomic influences on the sinoatrial node, and changes can be quantified as heart rate variability (HRV). In addition, after a premature ventricular beat, there are reproducible variations in RR interval, also due to baroreflex mediated autonomic influences on the sinoatrial node, that can be measured as heart rate turbulence (HRT). Impaired autonomic function as measured by HRV and HRT has proven to predict adverse outcomes in clinical settings. The ability of reduced HRV and HRT to predict adverse outcomes has been explained by their dependency on vagal mechanisms that could reflect an increased sympathetic and a reduced vagal modulation of sinus node, thus favoring cardiac electrical instability. Analysis of non-linear dynamics of HRV has also been utilized to describe the fractal like characteristic of the variability signal and proven effective in identify patients at risk for sudden cardiac death. Despite the clinical validity of these measures, it has also been evident that the relationship between neural input and sinus node responsiveness is extremely complex and variable in different clinical conditions. Thus, abnormal HRV or HRT on a clinical Holter recordings may reflect non-neural as well as autonomic mechanisms, and this also needs to be taken into account when interpreting any findings. However, under controlled conditions, the computation of the low and high frequency components of HRV and of their normalized powers or ratio seems capable of providing valid information on sympatho-vagal balance in normal subjects, as well as in most patients with a preserved left ventricular function. Thus, analysis of HRV does provide a unique tool to specifically assess autonomic control mechanisms in association with various perturbations. In conclusion, HRV measures are of substantial utility to identify patients with an increased cardiac mortality and to evaluate autonomic control mechanisms, but their ability to capture specific levels of autonomic control may be limited to controlled laboratory studies in relatively healthy subjects.

Keywords: autonomic modulation, sympathetic and vagal control, baroreflex mechanisms, spectral analysis, non-invasive evaluation of cardiac function

\section{HEART RATE VARIABILITY}

Heart rate variability (HRV) was the first non-invasive methodology extensively used to evaluate autonomic modulation of the sinus node in normal subjects and in patients with different cardiac and non-cardiac diseases and to identify patients at risk for an increased cardiac mortality. Since the initial report of Wolf et al. (1978) of the relationship of decreased RR variability on ECG and morality in post-MI patients, different methodologies have been developed and are now available to measure HRV in the experimental laboratory and in the clinical setting (Malik and Camm, 1995; Task Force of the European Society of Cardiology and the North American Society of Pacing and Electrophysiology, 1996). These range from simple statistical descriptors to complex nonlinear mathematical parameters. Indeed, the possibility of obtaining a non-invasive evaluation of autonomic control mechanisms has resulted in a relative broad utilization of this methodology, often in a "black box" manner, by people who have bought HRV software. However, a precise understanding of and a consensus on the autonomic underpinnings of most HRV parameters has not been achieved.

\section{TIME DOMAIN HRV PARAMETERS}

In almost all normal subjects and patients, the heart period changes on a beat to beat basis even during resting, controlled conditions. These variations, largely due to respiration and sympathetic activity are almost abolished by simultaneous parasympathetic and sympathetic blockade (Figure 1). When the recording period is prolonged to several hours and conditions are no longer tightly controlled, additional factors may influence HRV. Among important influences are: environmental factors, level and extent of physical activity, emotional stress, meal times, talking vs. silence, sleep and quality of sleep, and recording duration.

Overall variability is largely dependent on the strength of the circadian rhythm with a predominant sympathetic activity during day-time and a predominant vagal activity during night-time. These autonomic changes can be easily inferred by considering the 


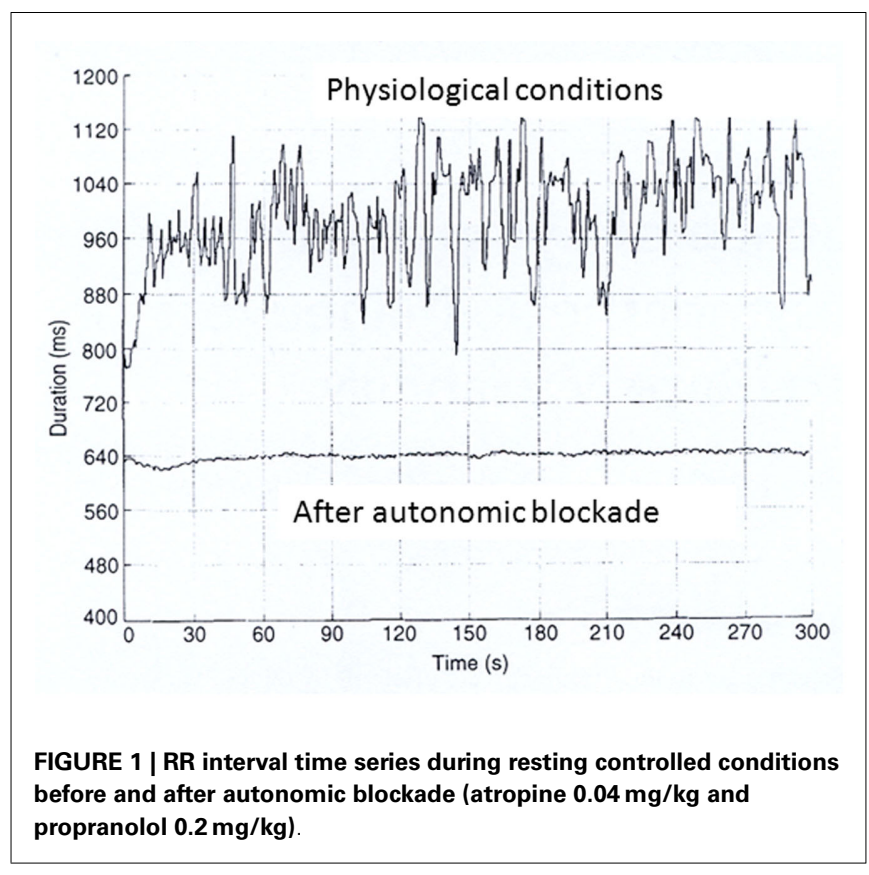

variation in $24 \mathrm{~h}$ heart rate patterns. A reduced SDNN or triangular index is generally due to failure of heart rate to decrease at night and reflects an impaired vagal activity. Moreover, an increased resting heart rate is often present in patients with sign of sympathetic activation. These autonomic alterations are made worse by low day-time activity levels. Thus, both a diminished vagal and an increased sympathetic modulation of the sinus node may be reflected by a reduction in HRV (Kleiger et al., 1987; Malik and Camm, 1995; Task Force of the European Society of Cardiology and the North American Society of Pacing and Electrophysiology, 1996; Fauchier et al., 1997; Nolan et al., 1999; Rashba et al., 2006). This interpretation is in agreement with experimental evidence indicating a pro-arrhythmic effect of sympatho-excitation (Lown and Verrier, 1976) and also with the findings that a reduction of these parameters is associated with an increased cardiac mortality in almost all clinical conditions characterized by an autonomic imbalance, e.g., after myocardial infarction, in patients with heart failure, hypertension, or diabetes (Kleiger et al., 1987; Malik and Camm, 1995; Task Force of the European Society of Cardiology and the North American Society of Pacing and Electrophysiology, 1996; Fauchier et al., 1997; Nolan et al., 1999; Rashba et al., 2006).

The finding that time domain parameters were inversely correlated with aging and influenced by the extent of left ventricular dysfunction made clear that the causes of reduction in HRV and the interpretation of their predictive value were more complex than originally assumed and also involved neural and non-neural mechanisms (Malik and Camm, 1995; Task Force of the European Society of Cardiology and the North American Society of Pacing and Electrophysiology, 1996).

Indeed, the relationship between neural discharge, heart rate, and HRV is complex and cannot be explained by a linear model. Experimental findings, indicate (Zaza and Lombardi, 2001) that neural modulation of cycle length is rather dependent on where on the curve they are, much as cardiac contractility is not uniform over different amounts of stretch of the cardiac muscle. Thus, although the finding of an intrinsic rate dependency of autonomic indices does not limit the prognostic value of HRV, it markedly complicates its physiological interpretation. Moreover, it has also to be considered that at the sinus node transduction level acetylcholine-mediated effects occur with a faster kinetics than those of norepinephrine, thus having a larger potential for inducing near-term variability. Although this forms the basis of measuring acetylcholine-mediated vagal control of heart rate by examining more rapid changes in cycle length, it also complicates the interpretation of slower changes. In addition two other factors need to be considered: first, an increase in resting heart period duration may amplify the effects of any source of variability affecting the sinus node and second, that many cardiovascular drugs may directly affect the sinus pacemaker. All these factors have to be taken into account when interpreting HRV findings (Malik and Camm, 1995; Task Force of the European Society of Cardiology and the North American Society of Pacing and Electrophysiology, 1996).

Time domain variables can be divided into indexes of total variability such as SDNN, SDANN, or triangular index, preferably computed on long term recordings, and beat to beat indexes such as rMSSD or pNN50 that are more specific markers of $\mathrm{vagal} /$ respiratory effects but are also exaggerated by subtle arrhythmias that are not of respiratory origin, especially in older populations or in subjects with underlying clinical disease. These latter parameters can also be assessed on short-term recordings and have been frequently used to evaluate alterations in vagal modulation, although unless the subjects being studied are young and healthy, verification, by using a graphical analysis, that the measures truly reflect respiratory sinus arrhythmia, is suggested (Stein et al., 2005b).

\section{FREOUENCY DOMAIN PARAMETERS}

Spectral analysis allows the identification and quantification of the principal oscillations that characterize HRV especially during resting controlled conditions. Since the beginning, interest in this approach was driven by the possibility of correlating shortterm spectral components to neural discharge (Akselrod et al., 1981; Pagani et al., 1986; Malliani et al., 1991; Task Force of the European Society of Cardiology and the North American Society of Pacing and Electrophysiology, 1996) and of obtaining indirect information on neural modulation of the sinus node. In normal subjects under controlled conditions (Figure 2), two principal components are easily identified: a respiration-related high frequency (HF) component $(0.15-0.4 \mathrm{~Hz})$ and a lower frequency (LF) component $(0.04-0.15 \mathrm{~Hz})$ with a peak at about $0.1 \mathrm{~Hz}$ synchronous with the low frequency oscillations of arterial pressure (Akselrod et al., 1981; Pagani et al., 1986; Malliani et al., 1991; Task Force of the European Society of Cardiology and the North American Society of Pacing and Electrophysiology, 1996). The former was considered to reflect phasic vagal activity triggered by respiration. The origin of LF was more controversial, being the result of more complex neural mechanisms related to sympathetic and vagal outflows. The LF/HF ratio has been used as an index of sympatho-vagal interaction to explore autonomic control of 


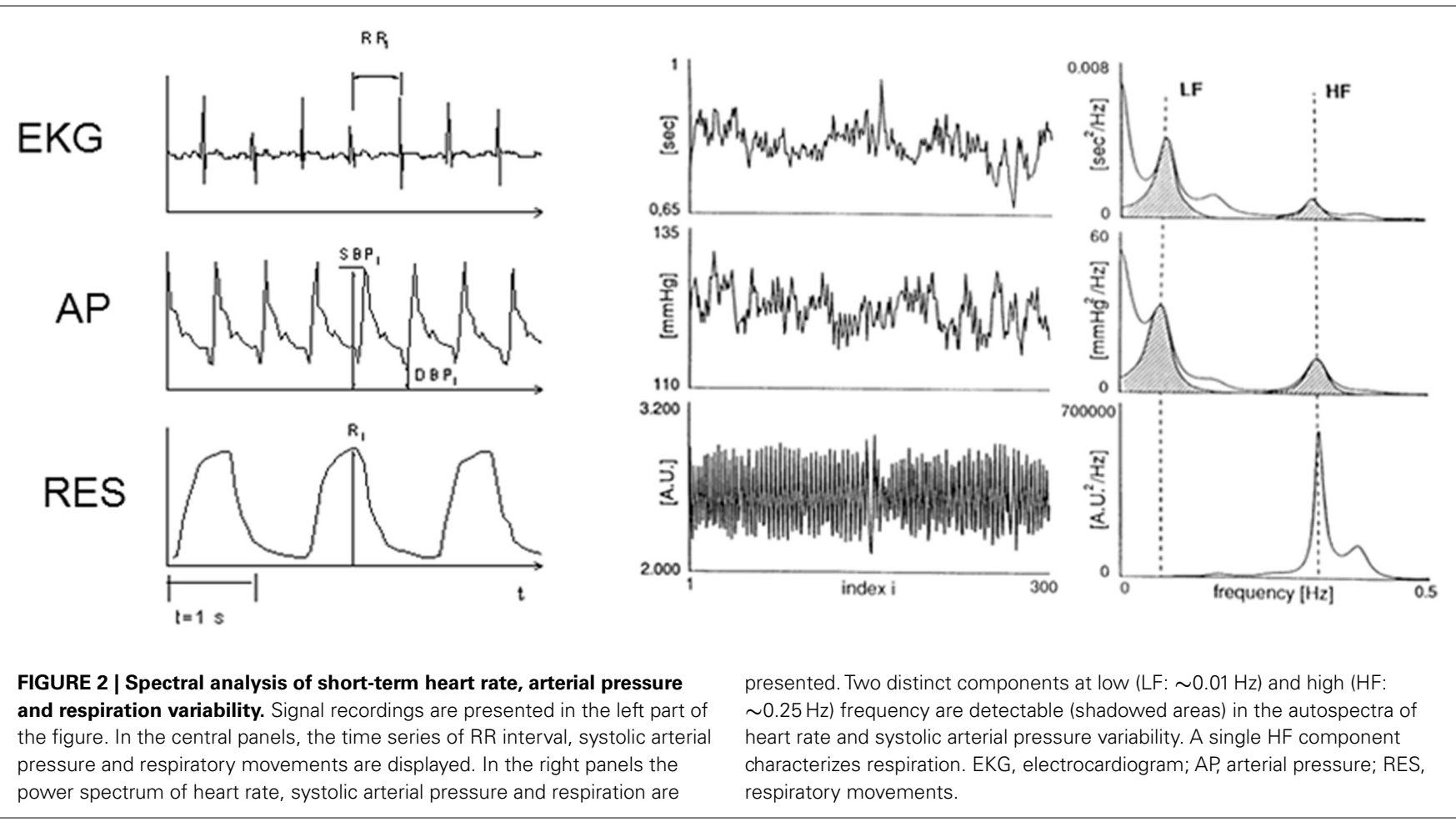

sinus node during experimental intervention capable of producing reflex sympathetic or vagal activation (Akselrod et al., 1981; Pagani et al., 1986; Malliani et al., 1991; Task Force of the European Society of Cardiology and the North American Society of Pacing and Electrophysiology, 1996). This was the case, for example, in patients during the acute phase of myocardial infarction or in the initial stages of heart failure (Akselrod et al., 1981; Pagani et al., 1986; Lombardi et al., 1987; Malliani et al., 1991; Task Force of the European Society of Cardiology and the North American Society of Pacing and Electrophysiology, 1996). In these patients, values of LF/HF ratio greater than 2 were considered to reflect a shift of sympatho-vagal balance toward a sympathetic predominance. More controversial was the interpretation of spectral component in patients with a marked reduction of ventricular function, where in spite of clinical signs of sympathetic activation, a reduction rather than an increase in LF component is commonly observed (van de Borne et al., 1997). Different factors including an altered central pattern of discharge at vasomotor centers, a loss of rhythmicity in sympathetic outflow as a consequence of a reduced baroreflex modulation, as well as a reduced responsiveness of the sinus node to neural inputs have been repeatedly advocated as explantation without, however, definitive experimental data (Malliani et al., 1991; Task Force of the European Society of Cardiology and the North American Society of Pacing and Electrophysiology, 1996; Burger et al., 1997; Grassi et al., 1999).

The issue of the meaning of LF, HF, or LF/HF is even more controversial when interpreting long term recordings (Figure 3) whereas environmental factors, extent of physical activity, and quality and duration of sleep are the major determinants of HRV and these components vary significantly over time (Malliani et al.,

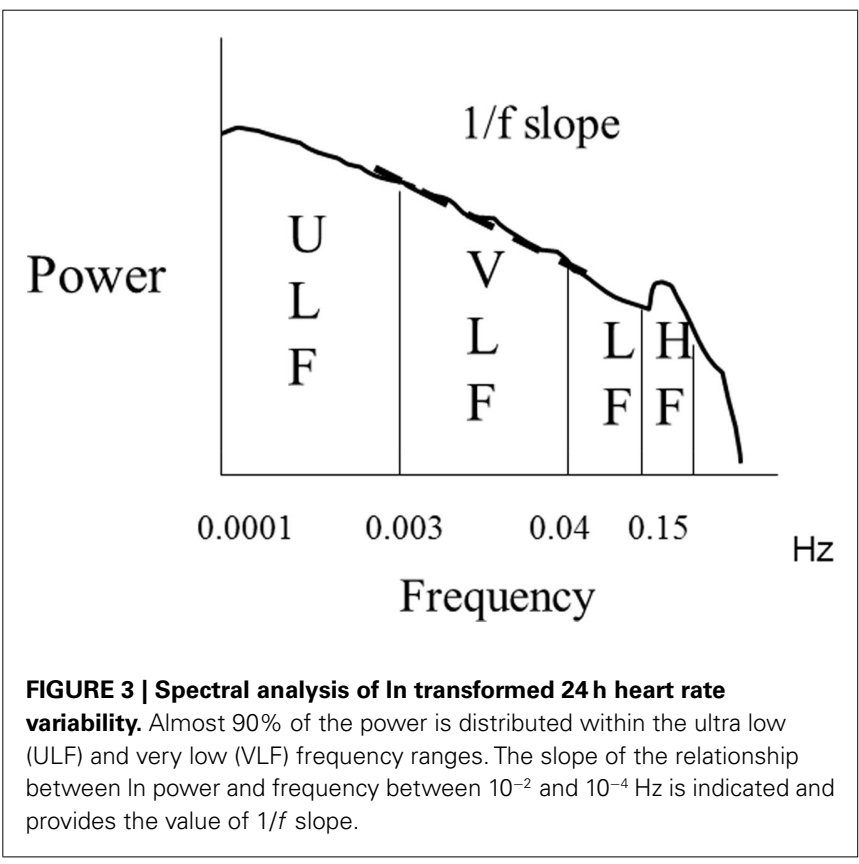

1991; Bigger et al., 1992; Task Force of the European Society of Cardiology and the North American Society of Pacing and Electrophysiology, 1996).

In conclusion, spectral analysis of HRV must be restricted to short-term recordings under controlled conditions in order to definitely relate them to autonomic nervous system functioning. However, just as ST depression on a resting ECG is useful for finding clinical problems, a more definitive stress test 
is needed to fully unmask ST depression. Similarly, short-term supine, controlled measures of HRV, which reflecting current autonomic status, may identify patients with extremely poor autonomic functioning but will miss autonomic abnormalities that are not present at rest. In addition, when associated with simultaneous continuous recording of arterial pressure and of respiratory activity (Pagani et al., 1986; Parati et al., 1988; Task Force of the European Society of Cardiology and the North American Society of Pacing and Electrophysiology, 1996), spectral analysis offers the unique opportunity of evaluating the effects of arterial pressure changes on heart period and to compute baroreflex sensitivity, an important marker of cardiovascular integration.

\section{NON-LINEAR DYNAMICS}

The appraisal of the complexity of the different neural and nonneural feedback loops impacting on the sinus node and determining HRV has stimulated the search for novel indexes capable of reflecting the complexity and the correlation properties of the signal rather than the magnitude of variability (Goldberger, 1996; Task Force of the European Society of Cardiology and the North American Society of Pacing and Electrophysiology, 1996). Indices such as the exponent $\beta$ of $1 / f$ slope for long term analysis (Goldberger, 1996; Task Force of the European Society of Cardiology and the North American Society of Pacing and Electrophysiology, 1996; Lombardi, 2000) or the scaling exponent $\alpha^{1}$ for short-term recordings (Peng et al., 1995; Mäkikallio et al., 2005) provide measures of fractal correlation properties of RR intervals at different time scales. These parameters do not reflect a specific component of autonomic modulation and therefore cannot be used to evaluate the presence of increased sympathetic activation or of a reduced vagal tone. They rather reflect the characteristics of heart rate behavior and, in particular, its complexity or randomness which are dependent upon the functional integrity of autonomic control mechanisms and sinus node responsiveness (Task Force of the European Society of Cardiology and the North American Society of Pacing and Electrophysiology, 1996).

In normal subjects the power-law slope has been reported to be equal to -1 , although this assertion is yet not supported by epidemiologic data. It becomes more negative after myocardial infarction (Task Force of the European Society of Cardiology and the North American Society of Pacing and Electrophysiology, 1996; Lombardi, 2000) and with aging (Stein et al., 2008). In ICD recipient patients, analysis of the storage electrograms revealed a further reduction of power-law slope of slow fluctuations in the minutes preceding the onset of ventricular tachycardia in comparison to control (Lombardi et al., 2000).

The detrended fluctuation analysis technique quantifies the relations of heart rate fluctuations at different time scales. In younger populations, the short-term fractal scaling exponent $\alpha^{1}$ (or DFA1; correlations on the scale of 3-11 beats) in the 1.20-1.35 range is commonly observed. In the Cardiovascular Health study (Stein et al., 2008), DFA1 of 1.05 was the cutpoint value for risk of mortality. Lower values in post-MI studies and in CHF patients $(<0.80$ or 0.85 , respectively) indentify patients at higher risk (Huikuri et al., 2000). The short-term fractal scaling exponent has been particularly effective in identifying patients at risk for sudden cardiac death (Task Force of the European Society of Cardiology and the North American Society of Pacing and Electrophysiology, 1996; Mäkikallio et al., 1999, 2005; Huikuri et al., 2000; Lombardi et al., 2001; Stein et al., 2005a, 2008). The analysis of short-term fractal properties of HRV has been demonstrated to be superior to conventional HRV measures in term of prognostic value in predicting both arrhythmic and non-arrhythmic cardiac death in post myocardial infarction patients (Mäkikallio et al., 2005). Longer term DFA without correction is not very useful for risk stratification and DFA2 $\left(\alpha^{2}\right.$, correlations on the scale of $12-20$ beats) has not been shown to add to risk stratification in most situations.

Altered short-term fractal scaling exponent has also been observed to precede ventricular fibrillation onset among patients who experienced this arrhythmia during Holter recordings (Mäkikallio et al., 1999).

\section{HEART RATE TURBULENCE}

Whereas alterations in RR intervals induced by premature ventricular beats can affect computation of time domain parameters and the LF and HF components by increasing, respectively, overall variability as well as power in the HF range (Malik and Camm, 1995; Task Force of the European Society of Cardiology and the North American Society of Pacing and Electrophysiology, 1996), they are the basis for heart rate turbulence (HRT) analysis.

This methodology, initially described by Schmidt et al. (1999), is based on the fact that the changes in RR intervals following a premature ventricular beat in healthy people are well-defined and consist of an initial shortening followed by a progressive lengthening until there is a return to the pre-arrhythmia baseline. This pattern of changes, although not necessarily the exact magnitude, is consistent within the same subject and is the result of the interaction of complex neural control mechanisms (Bauer et al., 2008).

\section{PHYSIOLOGICAL INTERPRETATIONS OF HRT}

Since the original description of HRT methodology (Schmidt et al., 1999; Bauer et al., 2008), it was hypothesized that the neural reflexes responsible for HRT were due to two principal components: the initial acceleration was the result of a transient vagal inhibition and sympathetic activation in response to brief inhibition of baroreflex afferent input due to a hemodynamically inefficient ventricular contraction (Lombardi et al., 1989; Schmidt et al., 1999; Davies et al., 2001; Bauer et al., 2008). This explanation proved to be incomplete. The initial HR acceleration is a fast response phenomenon thus making unlikely that a surge in sympathetic outflow could play a major role. Then, after the compensatory pause, it was hypothesized that the overshoot of arterial pressure, due to increased ventricular filling, was responsible for the subsequent deceleration of heart rate through a combined sympathetic and vagal recruitment. Thus two distinct phenomena characterize HRT: the initial shortening, which is defined by the term turbulence onset (TO) and the subsequent lengthening that is described by the term turbulence slope (TS; Figure 4). A more complete appraisal of HRT mechanisms, took in account the fact 

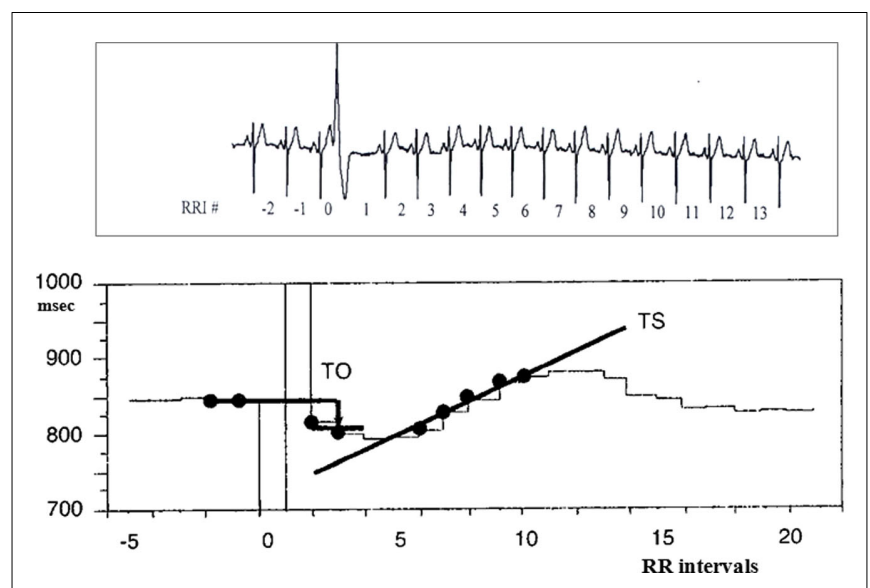

FIGURE 4 | Schematic representation of the RR interval changes induced by a premature ventricular contraction that are used to compute the two indexes of heart rate turbulence: turbulence onset (TO) and turbulence slope (TS).

that under physiologic conditions, systolic arterial pressure progressively increases after a premature ventricular beat and reaches its maximal value within eight cardiac cycles, thus activating a spontaneous baroreflex mechanism, with an initial sympathetic inhibition followed by a transient predominant vagal activation (Lombardi et al., 1989; Schmidt et al., 1999; Davies et al., 2001; Bauer et al., 2008). This explanation is supported by the finding that greatest variations in HRT indexes were observed in response to more premature spontaneous or induced ventricular contractions, which were associated with a greater fall in systolic pressure and longer compensatory pause (Davies et al., 2001; Roach et al., 2002; Watanabe et al., 2002; Savelieva et al., 2003; Wichterle et al., 2006; Bauer et al., 2008).

Heart rate turbulence is computed as TO and TS. TO is computed (Schmidt et al., 1999; Bauer et al., 2008) by determining the percentage differences between the two sinus $R R$ intervals following the compensatory pause $\left(\mathrm{RR}_{1}\right.$ and $\left.\mathrm{RR}_{2}\right)$ and the two $\mathrm{RR}$ intervals immediately preceding the premature beat coupling interval $\left(R_{-2}\right.$ and $\left.R R_{-1}\right)$ divided by $R_{-2}+R_{-1}$. The initial acceleration is therefore computed on the first two sinus cycles following the premature ventricular beat and if there is no acceleration or an actual deceleration, both abnormal, TO is zero or positive. TS is defined as the maximum regression slope measured on any 5 consecutive sinus cycles within the first 15 sinus intervals after the compensatory pause and cannot be calculated when there are fewer than 15 sinus beats after the premature ventricular contraction. The slowing is due to a baroreflex mediated vagal activation and it is expressed in ms/RR interval. Computation of HRT requires at least five qualifying ventricular premature beats and TO and TS are computed from a "signal average" of the responses to all premature ventricular beats on the recording.

Both TO and TS were found to correlate with baroreflex sensitivity assessed by the phenylephrine method. The physiological changes in sinus cycle duration measure by HRT were abolished by vagal blockade with atropine and were almost unaffected after beta-blockade (Bauer et al., 2008).
The correlation between HRT and HRV is modest, suggesting that additional neural and non-neural factors are likely to play a major role in determining variations in the parameters that describes the two methodologies and that there is potential to use them in combination to leverage the risk stratification properties of both. In support of this, addition, the use of a combination of a decreased short-term fractal scaling exponent and abnormal HRT identified population-dwelling older adults at higher risk of cardiovascular death than the use of either parameter alone.

\section{FACTORS INFLUENCING HRT}

Left ventricular ejection fraction significantly affects HRT parameters. In patients with structural heart disease and depressed left ventricular function, HRT indexes are markedly depressed as a result of mechanical and autonomic factors: the enhanced post-extrasystolic potentiation and the altered autonomic pattern with signs of sympathetic activation and reduced vagal modulation account for such a finding (Bauer et al., 2008). However, a recent study showed that abnormal values for HRT identify older adults who are considered to be healthy by multiple measures of cardiovascular function including HRV, but who were, in fact, at almost eight times greater risk of cardiovascular death on follow up than healthy older adults with normal HRT (Stein and Barzilay, 2011).

Aging is also associated with a reduction of HRT parameters in a manner similar to most autonomic indexes. Finally HRT is reduced at higher heart rate, as a result of either a baseline sympathetic activation or of a heart rate dependency associated with the non-linear relationship between sinus node properties and vagal neural activity (Schwab et al., 2004; Bauer et al., 2008).

\section{CONCLUSION}

Heart rate variability and HRT are two non-invasive ECG-based techniques capable of providing relevant information on the autonomic modulation of the sinus node. They can be considered as complementary, taking into account that HRV mainly reflects the continuous interaction between neural modulatory mechanism and sinus node function while HRT can be considered a physiological stimulus-response test in which sinus node function and autonomic modulatory activity are perturbed by a transient stimulus either occurring spontaneously or induced by programmed electric stimulation.

The predictive value of these methodologies will be discussed in the next section. Of clinical relevance is the finding that depressed values for both HRV and HRT indexes are commonly observed in those clinical conditions associated with an increased cardiovascular mortality (Task Force of the European Society of Cardiology and the North American Society of Pacing and Electrophysiology, 1996; Bauer et al., 2008, 2009). Finally, the association between a reduced SDNN and markers of subclinical inflammation suggests that reduced vagal activity may mediate inflammation which opens new perspectives, not only for a better understanding on the physiopathological mechanisms responsible for cardiac mortality, but also for the identification of high-risk patients by combining autonomic with inflammatory markers (Lombardi, 2004; Stein and Barzilay, 2011). 


\section{REFERENCES}

Akselrod, S., Gordon, D., Ubel, F. A., Shannon, D. C., Barger, A. C., and Cohen, R. J. (1981). Power spectrum analysis of heart rate fluctuation: a quantitative probe of beat-to-beat cardiovascular control. Science 213, 220-222.

Bauer, A., Barthel, P., Müller, A., Ulm, K., Huikuri, H., Malik, M., and Schmidt, G. (2009). Risk prediction by heart rate turbulence and deceleration capacity in postinfarction patients with preserved left ventricular function retrospective analysis of 4 independent trials. J. Electrocardiol. 42, 597-601.

Bauer, A., Malik, M., Schmidt, G., Barthel, P., Bonnemeier, H., Cygankiewicz, I., Guzik, P., Lombardi, F., Müller, A., Oto, A., Schneider, R., Watanabe, M., Wichterle, D., and Zareba, W. (2008). Heart rate turbulence: standards of measurement, physiological interpretation, and clinical use international society for Holter and noninvasive electrophysiology consensus. J. Am. Coll. Cardiol. 52, 1353-1365.

Bigger, J. T., Fleiss, J., Steinman, R. C., Rolnitzky, L. M., Kleiger, R. E., and Rottman, J. N. (1992). Frequency domain measures of heart period variability and mortality after myocardial infarction. Circulation 85, 164-171.

Burger, A. J., Charlamb, M., Weinrauch, L. A., and D'Elia, J. A. (1997). Short, and long-term reproducibility of heart rate variability in patients with long-standing type I diabetes mellitus. Am. J. Cardiol. 80, 1198-1202.

Davies, L. C., Francis, D. P., Ponikowski, P., Piepoli, M. F., and Coats, A. J. (2001). Relation of heart rate and blood pressure turbulence following premature ventricular complexes to baroreflex sensitivity in chronic congestive heart failure. Am. J. Cardiol. 87, 737-742.

Fauchier, L., Babutj, D., Cosnay, P., Autret, M. L., and Fauchier, J. P. (1997). Heart rate variability in idiopathic dilated cardiomyopathy characteristics and prognostic value. J. Am. Coll. Cardiol. 30, 1009-1014.

Goldberger, A. L. (1996). Non-linear dynamics for clinicians: chaos theory, fractals, and complexity at the bedside. Lancet 347, 1312-1314.

Grassi, G., Turri, C., Vailati, S., Dell'Oro, R., and Mancia, G. (1999). Muscle and skin sympathetic nerve traffic during the "white-coat" effect. Circulation 100, 222-225.

Huikuri, H. V., Makikallio, T. H., Peng, C. K., Golberger, A. L., Hintze, U.,
Moller, M., and for the Diamond Study Group. (2000). Fractal correlation properties of R-R interval dynamics and mortality in patients with depressed left ventricular function after an acute myocardial infarction. Circulation 101, 47-53.

Kleiger, R. E., Miller, J. P., Bigger, J. T., Moss, A. R., and Multicenter PostInfarction Research Group. (1987). Decreased heart rate variability and its association with increased mortality after acute myocardial infarction. Am. J. Cardiol. 59, 256-262.

Lombardi, F. (2000). Chaos theory, heart rate variability and arrhythmic mortality. Circulation 101, 8-10.

Lombardi, F. (2004). Sympathetic activation and subclinical inflammation: a new combination to identify high risk subjects. Eur. Heart J. 25, 359-360.

Lombardi, F., Gnecchi Ruscone, T., and Malliani, A. (1989). Premature ventricular contractions and reflex sympathetic activation in cats. Cardiovasc. Res. 23, 205-212.

Lombardi, F., Makikallio, T. H., Myerburg, R. J., and Huikuri, H. V. (2001). Sudden cardiac death: role of heart rate variability to identify patients at risk. Cardiovasc. Res. 50, 210-217.

Lombardi, F., Porta, A., Marzegalli, M., Favale, S., Santini, M., Vincenti, A., and De Rosa, A. (2000). Heart rate variability patterns before ventricular tachycardia onset in patients with implantable cardioverter defibrillator. Am. J. Cardiol. 86, 959-963.

Lombardi, F., Sandrone, G., Pernpruner, S., Sala, R., Garimoldi, M., Cerutti, S., Baselli, G., Pagani, M., and Malliani, A. (1987). Heart rate variability as an index of sympathovagal interaction after acute myocardial infarction. Am. J. Cardiol. 60, 1239-1245.

Lown, B., and Verrier, R. L. (1976). Neural activity and ventricular fibrillation. N. Engl. J. Med. 294, 1165-1176.

Mäkikallio, T. H., Barthel, P., Schneider, R., Bauer, A., Tapanainen, J. M., Tulppo, M. P., Schmidt, G., and Huikuri, H. V. (2005). Prediction of sudden cardiac death after acute myocardial infarction: role of Holter monitoring in the modern treatment era. Eur. Heart J. 26, 762-769.

Mäkikallio, T. H., Koistinen, J., Jordaens, L., Tulppo, M. P., Wood, N., Glosarsky, B., Peng, C. K., Goldberger, A. L., and Huikuri, H. V. (1999). Heart rate dynamics before spontaneous onset of ventricular fibrillation in patients with healed myocardial infarcts. Am. J. Cardiol. $83,880-884$.
Malik, M., and Camm, A. J. (1995). Heart Rate Variability. Armonk, NY: Futura Publishing Company.

Malliani, A., Pagani, M., Lombardi, F., and Cerutti, S. (1991). Cardiovascular neural regulation explored in the frequency domain. Circulation 84, 482-492.

Nolan, J., Batin, P. D., and Andrews, R. (1999). Prospective study of heart rate variability and mortality in chronic heart failure: results of the United Kingdom Heart Failure Evaluation and Assessment of Risk Trial (UK-Heart). Circulation 98, 1510-1516.

Pagani, M., Lombardi, F., Guzzetti, S., Rimoldi, O., Furlan, R., Pizzinelli, P., Sandrone, G., Malfatto, G. Dell'Orto, S., Piccaluga, E., Turiel, M., Baselli, G., Cerutti, S., and Malliani, A. (1986). Power spectral analysys of heart rate and arterial pressure variabilities as a marker of sympathovagal interaction in man and consciuos dog. Circ. Res. 59, 178-193.

Parati, G., Di Rienzo, M., and Bertinieri, G. (1988). Evaluation of the baroreceptor heart rate reflex by 24 hours intra-arterial blood pressure monitoring in humans. Hypertension 12, 214-222.

Peng, C. K., Havlin, S., Stanley, H. E., and Goldberger, A. L. (1995). Quantification of scaling exponents and crossover phenomena in nonstationary heartbeat time series. Chaos 5, 82-87.

Rashba, E. J., Estes, N. A., Wang, P., Schaechter, A., Howard, A., Zareba, W., Couderc, J. P., Perkiomaki, J., Levine, J., and Kadish, A. (2006). Preserved heart rate variability identifies low-risk patients with nonischemic dilated cardiomyopathy: results from the DEFINITE trial. Heart Rhythm 3, 281-286.

Roach, D., Koshman, M. L., Duff, H., and Sheldon, R. (2002). Induction of heart rate and blood pressure turbulence in the electrophysiologic laboratory. Am. J. Cardiol. 90, 1098-1102.

Savelieva, I., Wichterle, D., Harries, M., Meara, M., Camm, A. J., and Malik, M. (2003). Heart rate turbulence after atrial, and ventricular premature beats: relation to left ventricular function, and coupling intervals. Pacing Clin. Electrophysiol. 26, 401-405.

Schmidt, G., Malik, M., Barthel, P., Schneider, R., Ulm, K., Rolnitzky, L., Camm, A. J., Bigger, J. T. Jr., and Schomig, A. (1999). Heart-rate turbulence after ventricular premature beats as a predictor of mortality after acute myocardial infarction. Lancet 353, 1390-1396.

Schwab, J. O., Eichner, G., Veit, G., Schmitt, H., Lewalter, T., and Luderitz, B. (2004). Influence of basic heart rate and sex on heart rate turbulence in healthy subjects. Pacing Clin. Electrophysiol. 27, 1625-1631.

Stein, P. K., and Barzilay, J. I. (2011). Relationship of abnormal heart rate turbulence and elevated CRP to cardiac mortality in low, intermediate, and high-risk older adults. J. Cardiovasc. Electrophysiol. 22, 122-127.

Stein, P. K., Barzilay, J. I., Chaves, P. H., Mistretta, S. Q., Domitrovich, P. P., Gottdiener, J. S., Rich, M. W., and Kleiger, R. E. (2008). Novel measures of heart rate variability predict cardiovascular mortality in older adults independent of traditional cardiovascular risk factors: the Cardiovascular Health Study (CHS). J. Cardiovasc. Electrophysiol. 19, 1169-1174.

Stein, P. K., Domitrovich, P. P., Huikuri, H. V., Kleiger, R. E., and Cast Investigators. (2005a). Traditional and nonlinear heart rate variability are each independently associated with mortality after myocardial infarction. J. Cardiovasc. Electrophysiol. 16, 13-20.

Stein, P. K., Domitrovich, P. P., Hui, N., Rautaharju, P., and Gottdiener, J. (2005b). Sometimes higher heart rate variability is not better heart rate variability: results of graphical and nonlinear analyses. J. Cardiovasc. Electrophysiol. 16, 954-959.

Task Force of the European Society of Cardiology and the North American Society of Pacing and Electrophysiology. (1996). Heart rate variability. Standards of measurement, physiological interpretation, and clinical use. Circulation 93, 1043-1065.

van de Borne, P., Montano, N., Pagani, M., Oren, R., and Somers, V. K. (1997). Absence of low-frequency variability of sympathetic nerve activity in severe heart failure. Circulation 95, 1449-1454.

Watanabe, M. A., Marine, J. E., Sheldon, R., and Josephson, M. E. (2002). Effects of ventricular premature stimulus coupling interval on blood pressure and heart rate turbulence. Circulation 106, 325-330.

Wichterle, D., Melenovsky, V., Simek, J., and Malik, M. (2006). Hemodynamics and autonomic control of heart rate turbulence. J. Cardiovasc. Electrophysiol. 17, 286-291.

Wolf, M. M., Varigos, G. A., Hunt, D., and Sloman, J. G. (1978). Sinus arrhythmia in acute myocardial infarction. Med. J. Aust. 2, 52-53. 
Zaza, A., and Lombardi, F. (2001). Autonomic indexes based on the analysis of heart rate variability: a view from the sinus node. Cardiovasc. Res. 50, $34-42$.

Conflict of Interest Statement: The authors declare that the research was conducted in the absence of any commercial or financial relationships that could be construed as a potential conflict of interest.

Received: 21 September 2011; paper pending published: 04 October 2011; accepted: 22 November 2011; published online: 08 December 2011.
Citation: Lombardi $F$ and Stein PK (2011) Origin of heart rate variability and turbulence: an appraisal of autonomic modulation of cardiovascular function. Front. Physio. 2:95. doi: 10.3389/fphys.2011.00095

This article was submitted to Frontiers in Clinical and Translational Physiology, a specialty of Frontiers in Physiology.
Copyright (C) 2011 Lombardi and Stein. This is an open-access article distributed under the terms of the Creative Commons Attribution Non Commercial License, which permits noncommercial use, distribution, and reproduction in other forums, provided the original authors and source are credited. 